University of Nebraska - Lincoln

DigitalCommons@University of Nebraska - Lincoln

Faculty Publications, Department of Physics and Astronomy

Research Papers in Physics and Astronomy

3-23-2005

\title{
Magnetoelectric Switching of Exchange Bias
}

\author{
Pavel Borisov \\ Universität Duisburg-Essen, p.borisov@lboro.ac.uk \\ Andreas Hochstrat \\ Universität Duisburg-Essen \\ Xi Chen \\ Universität Duisburg-Essen \\ Wolfgang Kleeman \\ Universität Duisburg-Essen, wolfgang.kleemann@uni-due.de \\ Christian Binek \\ University of Nebraska-Lincoln, cbinek@unl.edu
}

Follow this and additional works at: https://digitalcommons.unl.edu/physicsfacpub

Part of the Physics Commons

Borisov, Pavel; Hochstrat, Andreas; Chen, Xi; Kleeman, Wolfgang; and Binek, Christian, "Magnetoelectric Switching of Exchange Bias" (2005). Faculty Publications, Department of Physics and Astronomy. 22. https://digitalcommons.unl.edu/physicsfacpub/22

This Article is brought to you for free and open access by the Research Papers in Physics and Astronomy at DigitalCommons@University of Nebraska - Lincoln. It has been accepted for inclusion in Faculty Publications, Department of Physics and Astronomy by an authorized administrator of DigitalCommons@University of Nebraska Lincoln. 


\title{
Magnetoelectric Switching of Exchange Bias
}

\author{
Pavel Borisov, ${ }^{1}$ Andreas Hochstrat, ${ }^{1}$ Xi Chen, ${ }^{1}$ Wolfgang Kleemann, ${ }^{1, *}$ and Christian Binek ${ }^{2}$ \\ ${ }^{1}$ Angewandte Physik, Universität Duisburg-Essen, D-47048 Duisburg, Germany \\ ${ }^{2}$ Department of Physics and Astronomy, Ferguson Hall, University of Nebraska, Lincoln, Nebraska 68588-0111, USA
}

(Received 7 January 2005; published 23 March 2005)

The perpendicular exchange bias field, $H_{\mathrm{EB}}$, of the magnetoelectric heterostructure $\mathrm{Cr}_{2} \mathrm{O}_{3}(111) /(\mathrm{Co} / \mathrm{Pt})_{3}$ changes sign after field cooling to below the Néel temperature of $\mathrm{Cr}_{2} \mathrm{O}_{3}$ in either parallel or antiparallel axial magnetic and electric freezing fields. The switching of $H_{\mathrm{EB}}$ is explained by magnetoelectrically induced antiferromagnetic single domains which extend to the interface, where the direction of their end spins controls the sign of $H_{\mathrm{EB}}$. Novel applications in magnetoelectronic devices seem possible.

DOI: 10.1103/PhysRevLett.94.117203

The linear magnetoelectric (ME) effect has been proposed decades ago to realize the control of electric and magnetic polarization properties by complementary fields in possible applications [1]. However, appropriate materials, which might fulfill the technologic demands, only recently became available. In particular, multiferroic manganites show convincing switching properties of their ferroelectric polarization by a magnetic field (e.g., $\mathrm{TbMnO}_{3}$ [2] or $\mathrm{TbMn}_{2} \mathrm{O}_{5}$ [3]) or of their ferromagnetic magnetization by an electric field (e.g., $\mathrm{HoMnO}_{3}$ [4]). Their very applicability, however, remains limited, since their ME properties are typical low temperature features.

A more favorable situation is met for the archetypical ME material chromium oxide, $\mathrm{Cr}_{2} \mathrm{O}_{3}$, which becomes magnetoelectric above room temperature, viz. below its antiferromagnetic (AFM) Néel temperature, $T_{\mathrm{N}}=307 \mathrm{~K}$ [5]. This makes it interesting for devices involving the well-known exchange bias (EB) effect of exchange coupled ferromagnetic (FM) and AFM heterostructures [6]. They are widely used in magnetic random access memory cells or giant magnetoresistive read-heads [7]. EB denotes the horizontal shift of the FM hysteresis loop after proper magnetic field cooling (MFC) to below the ordering temperature of the AFM component, $T_{\mathrm{N}}$. In this Letter we present a novel ME based switching mechanism for the EB field, $H_{\mathrm{EB}}$, which may be envisaged for applications. The mechanism is based on the so-called ME field cooling (MEFC) process, which favors the growth of a distinct AFM single domain and thus an efficient control of the AFM interface moment whose sign is decisive for that of $H_{\mathrm{EB}}$.

In accordance with the phenomenological approach of Meiklejohn and Bean (MB) [6] there is now unanimity that a net interface magnetization of the antiferromagnet is necessary for generating EB. The simple MB expression

$$
\mu_{0} H_{\mathrm{EB}}=-J S_{\mathrm{AFM}} S_{\mathrm{FM}} /\left(M_{\mathrm{FM}} t_{\mathrm{FM}}\right)
$$

describes the dependence of the bias field $\mu_{0} H_{\mathrm{EB}}$ on a phenomenological coupling, $J$, between the FM and AFM interface magnetizations $S_{\mathrm{FM}}$ and $S_{\mathrm{AFM}}$, respectively,
PACS numbers: 75.80.+q, 75.50.Ee, $75.70 . \mathrm{Cn}, 75.70 . \mathrm{Kw}$

where $t_{\mathrm{FM}}$ and $M_{\mathrm{FM}}$ are the thickness and the saturation magnetization of the FM layer. Equation (1) suggests that an extrinsic control of the EB field can be achieved by modifying the value of $S_{\mathrm{AFM}}$. Suitable control parameters are, e.g., temperature and magnetic freezing field [8]. Less well-known is the control of $S_{\mathrm{AFM}}$ using the piezomagnetic effect as observed, e.g., in $\mathrm{Fe}_{0.6} \mathrm{Zn}_{0.4} \mathrm{~F}_{2} / \mathrm{Fe}$ [9]. Here we propose to achieve this goal by making use of a $\mathrm{ME}$ antiferromagnet, which allows to control interface magnetic moments by application of an external electric field [10].

Our specimen was the layered heterostructure $\mathrm{Cr}_{2} \mathrm{O}_{3}(111) / \mathrm{Pt} 0.5 \mathrm{~nm} /[\mathrm{Co} 0.3 \mathrm{~nm} / \mathrm{Pt} 1.5 \mathrm{~nm}]_{3} / \mathrm{Pt} 1.5 \mathrm{~nm}$, where the $\mathrm{Co} / \mathrm{Pt}$ multilayer yields perpendicular EB [11] when being coupled to the uniaxial [111] directed AFM order parameter of $\mathrm{Cr}_{2} \mathrm{O}_{3}$. The $\mathrm{Co} / \mathrm{Pt}$ multilayer is grown under UHV conditions on top of the (111) surface of a $\mathrm{Cr}_{2} \mathrm{O}_{3}$ single crystal with thickness $0.7 \mathrm{~mm}$ (MaTeck, Jülich). The latter was polished to optical flatness with $0.1 \mu \mathrm{m}$ diamond paste before transferring into the UHV chamber, where it was annealed for $3 \mathrm{~h}$ at $720 \mathrm{~K}$. The subsequent growth of the $\mathrm{Co} / \mathrm{Pt}$ multilayer took place at $500 \mathrm{~K}$ by thermal and $e$-beam evaporation with growth rates of $8 \times 10^{-3}$ and $1 \times 10^{-2} \mathrm{~nm} / \mathrm{s}$ for $\mathrm{Co}$ and $\mathrm{Pt}$, respectively. The growth of the multilayer was started with a Pt buffer layer of $0.5 \mathrm{~nm}$ thickness in order to avoid surface reactions of Co with oxygen (from the $\mathrm{Cr}_{2} \mathrm{O}_{3}$ crystal) into AFM $\mathrm{CoO}$ [12], and thus prevent additional $\mathrm{EB}$ due to the $\mathrm{CoO} / \mathrm{Co}$ interface [6].

The magnetic characterization of the samples was performed in a self-built liquid nitrogen cryostat equipped with an electromagnet in Faraday configuration using the magneto-optical Kerr effect in back-reflection geometry at a light wavelength $\lambda=670 \mathrm{~nm}$. The hysteresis loops were 40-60 times averaged in order to minimize noise. By this procedure equilibrium data beyond possible initial training effects [8] emerge. Figure 1 shows the EB effect as induced by conventional MFC in the presence of a [111] directed magnetic freezing field $\mu_{0} H_{\mathrm{fr}}=0.6 \mathrm{~T}$, from 350 to $298 \mathrm{~K}$, i.e., from above to below $T_{\mathrm{N}}$ (curve 1). The loop is fairly 


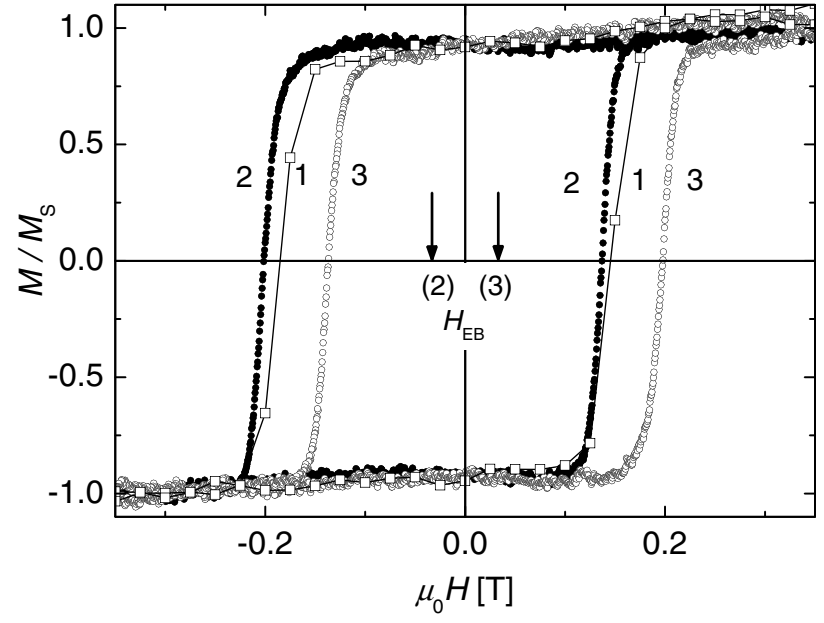

FIG. 1. Normalized hysteresis curves of $\mathrm{Cr}_{2} \mathrm{O}_{3}(111) /$ Pt $0.5 \mathrm{~nm} /[\text { Co } 0.3 \mathrm{~nm} / \mathrm{Pt} 1.5 \mathrm{~nm}]_{3} / \mathrm{Pt} 1.5 \mathrm{~nm}$ measured after magnetic field cooling in $\mu_{0} H_{\mathrm{fr}}=0.6 \mathrm{~T}$ and $E_{\mathrm{fr}}=0$ from $T=$ 350 to $298 \mathrm{~K}$ (1) and after magnetoelectric field cooling to $250 \mathrm{~K}$ in $\mu_{0} H_{\mathrm{fr}}=0.6 \mathrm{~T}$ and $E_{\mathrm{fr}}=-500 \mathrm{kV} / \mathrm{m}$ (2) and $E_{\mathrm{fr}}=$ $+500 \mathrm{kV} / \mathrm{m}$ (3), respectively. The lines are to guide the eyes. The exchange bias fields $\mu_{0} H_{\mathrm{EB}}$ referring to the loops 2 and 3 are indicated by arrows.

rectangular with its center shifted to negative fields by an EB field, $\mu_{0} H_{\mathrm{EB}}=-19.8 \mathrm{mT}$. A very similar hysteresis loop as shown by curve 2 with a negative EB shift, $\mu_{0} H_{\mathrm{EB}}=-32.1 \mathrm{mT}$, emerging after negative MEFC, i.e., under the simultaneous application of an electric field antiparallel to the magnetic field. Here the sample was cooled from $T=350$ to $250 \mathrm{~K}$ under the action of the freezing fields $\mu_{0} H_{\mathrm{fr}}=0.6 \mathrm{~T}$ and $E_{\mathrm{fr}}=-500 \mathrm{kV} / \mathrm{m}$. Surprisingly, when inverting the sign of $E_{\mathrm{fr}}$, i.e., when applying positive MEFC with $\mu_{0} H_{\mathrm{fr}}=0.6 \mathrm{~T}$ and $E_{\mathrm{fr}}=$ $+500 \mathrm{kV} / \mathrm{m}$, the $\mathrm{EB}$ also turns positive, $\mu_{0} H_{\mathrm{EB}}=$ $+30.3 \mathrm{mT}$ (curve 3 ). The sign obviously follows the inversion of the electric field from $E_{\mathrm{fr}}<0$ to $E_{\mathrm{fr}}>0$, while the sign of $\mu_{0} H_{\mathrm{fr}}$ has remained unchanged.

This amazing effect can be understood in terms of the so-called "magnetoelectric annealing" upon MEFC, which is known to create a single domain AFM state of the $\mathrm{Cr}_{2} \mathrm{O}_{3}$ crystal [13]. The two possible AFM domains $A$ and $B$, say, differ by opposite orientations of the AFM vector, $\mathbf{l}=\mathbf{s}_{1}-\mathbf{s}_{2}+\mathbf{s}_{3}-\mathbf{s}_{4}$, where 1-4 denote adjacent spins within the magnetic unit cell as shown schematically in the lower panels of Fig. 2(a) and 2(b) [14]. In simultaneously applied fields $H_{\mathrm{fr}}$ and $E_{\mathrm{fr}}$ they have different ME energies, $W_{\mathrm{ME}}=-2 \alpha_{z z} \mu_{0} H_{\mathrm{fr}} E_{\mathrm{fr}}$, where $\alpha_{z z}$ is the appropriate diagonal component of the magnetoelectric susceptibility tensor and $\alpha_{z z} \lessgtr 0$ refers to $A$ and $B$, respectively [15]. AFM single domaining under MEFC is, hence, due to the energy difference between $A$ and $B$ domains. The formation of domain $A$ upon cooling to below $T_{\mathrm{N}}$ is more probable than that of domain $B$ if $H_{\mathrm{fr}} E_{\mathrm{fr}}<0$, and vice versa for $H_{\mathrm{fr}} E_{\mathrm{fr}}>0$.

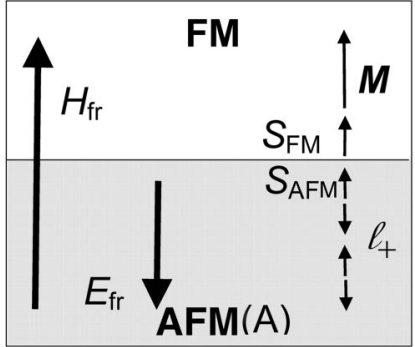

(a)

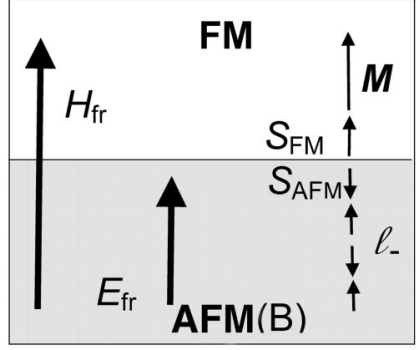

(b)
FIG. 2. Schematic sketches of a FM/AFM bilayer with freezing fields $\left(H_{\mathrm{fr}}, \mp E_{\mathrm{fr}}\right)$, order parameters $\left(\mathbf{M}, \mathbf{l}_{ \pm}\right)$, and magnetic moments $\left(S_{\mathrm{FM}}, S_{\mathrm{AFM}}\right)$, where the AFM layer is single domained ( $A$ and $B$, respectively) after MEFC (see text).

Bulk single domains are most convincingly demonstrated by their inherent ME susceptibility [5]. A net magnetic moment, $\Delta m \propto \alpha_{z z} E$, is induced along the $z$ axis by an external electric field, $E$, provided that the AFM system is single domained. This is shown in Fig. 3, where the magnetic moment of another heterostructure $\mathrm{Cr}_{2} \mathrm{O}_{3}(111) /(\mathrm{Co} / \mathrm{Pt})_{3}$ [16] has been measured by using superconducting quantum interference device (SQUID) magnetometry at $T=150 \mathrm{~K}$ in fields within the range $|E| \lesssim 400 \mathrm{kV} / \mathrm{m}$. While MFC of this sample in $\mu_{0} H_{\mathrm{fr}}=$ $0.5 \mathrm{~T}$ and $E_{\mathrm{fr}}=0$ gives rise to a multidomain state with nearly vanishing slope (line $1, \alpha_{z z} \approx 0$ ), MEFC with $\mu_{0} H_{\mathrm{fr}}=0.5 \mathrm{~T}$ and $E_{\mathrm{fr}}=-460$ and $+425 \mathrm{kV} / \mathrm{m}$ yields slopes of either sign, $\alpha_{z z}<0$ (line 2) and $\alpha_{z z}>0$ (line 3), due to the $A$ and $B$ domains, respectively. Note that the observed ME moments $\Delta m$ add to the remanence of the sample, $m(E=0) \approx 1.8 \times 10^{-8} \mathrm{~A} \mathrm{~m}^{2}$.

Obviously AFM single domain formation under MEFC is at the heart of the observed switching of $H_{\mathrm{EB}}$. First of all we have to consider that merely $1 \%-5 \%$ of the uncompensated AFM spins residing in the interface with the adjacent FM layer are responsible for the EB via $S_{\mathrm{AFM}}$ [17]. At $T<T_{\mathrm{N}}$ they occupy privileged positions, since they are robust against magnetic field cycles, which rotate the spins in both the FM and the topmost layers of the AFM component [18]. They are only loosely coupled to the FM, but strongly to the bulk of the AFM component. Hence, when inverting the orientation of $\mathbf{I}$ by MEFC, the orientation of the uncompensated spins, which constitute $S_{\mathrm{AFM}}$, will also be inverted. This is schematically depicted in Fig. 2 for the cases $\mu_{0} H_{\mathrm{fr}} E_{\mathrm{fr}}<0$ [2(a)] and $\mu_{0} H_{\mathrm{fr}} E_{\mathrm{fr}}>$ 0 [2(b)], where the rhombohedral unit cells within the two domains of $\mathrm{Cr}_{2} \mathrm{O}_{3}$ have either outward $(A)$ or inward directed $(B)$ end spins.

It is now tempting to assume that these end spins are mainly responsible for the interface coupling. This immediately implies that $S_{\mathrm{AFM}}$ and $S_{\mathrm{FM}}$ are either parallel or antiparallel to each other, thus giving rise to (conventional) negative or (unconventional) positive EB as observed. Note 


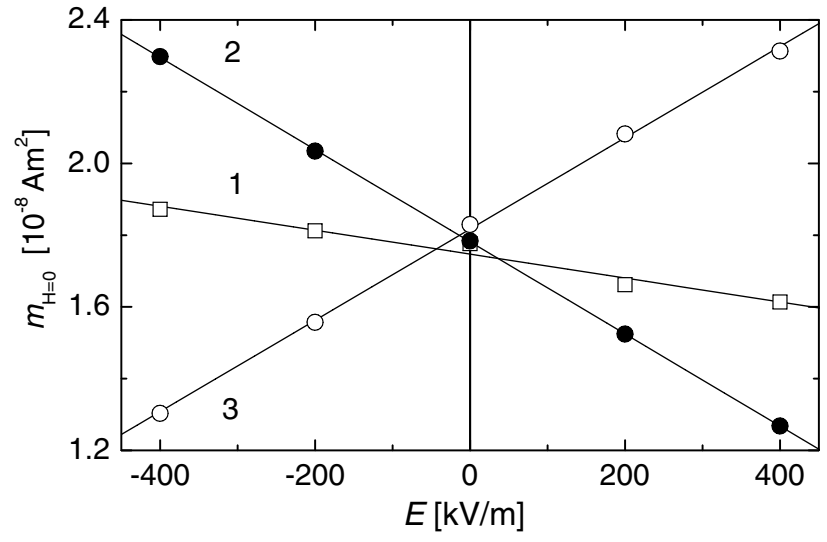

FIG. 3. Magnetic moment of $\mathrm{Cr}_{2} \mathrm{O}_{3}(111) / \mathrm{Pt} 0.7 \mathrm{~nm} /$ [Co $0.3 \mathrm{~nm} / \mathrm{Pt} 1.2 \mathrm{~nm}]_{3} / \mathrm{Pt} 3.1 \mathrm{~nm}$ in its remanent state, $m_{r}=$ $1.8 \times 10^{-8} \mathrm{~A} \mathrm{~m}^{2}$, as a function of the applied electric field $E$ at $T=150 \mathrm{~K}$ after MEFC from $T=350$ to $150 \mathrm{~K}$ in $\mu_{0} H_{\mathrm{fr}}=$ $0.5 \mathrm{~T}$ and $E_{\mathrm{fr}}=0$ (line 1 ), $-460 \mathrm{kV} / \mathrm{m}$ (line 2) and $+425 \mathrm{kV} / \mathrm{m}$ (line 3 ).

that this mechanism does not care about interface roughness. Its only prerequisite is the existence of complete AFM unit cells adjacent to the FM interface. Remarkably, the positive EB fields are systematically smaller by about $10 \%$ than the magnitudes of the negative ones. This appears reasonable when taking into account that the novel ME based coupling counteracts the conventional one for the case $\mu_{0} H_{\mathrm{fr}} E_{\mathrm{fr}}>0$. In that case some uncompensated pinned spins, which are less tightly bound to the AFM bulk or even dangling, will rather follow the FM alignment and favor negative EB.

Let us first mention a fundamental test of our hypothesis, which states that merely the sign of the product $H_{\mathrm{fr}} E_{\mathrm{fr}}$, and thus the kind of AFM domain, $A$ or $B$, decides the sign of $H_{\mathrm{EB}}$. To this end we have chosen all four sign combinations of the freezing fields with the magnitudes $\left|\mu_{0} H_{\mathrm{fr}}\right|=0.6 \mathrm{~T}$ and $\left|E_{\mathrm{fr}}\right|=500 \mathrm{kV} / \mathrm{m}$ for MEFC from $T=350$ to $298 \mathrm{~K}$. Evaluation of the hysteresis loops measured at $298 \mathrm{~K}$ yields the following shifts: $\mu_{0} H_{\mathrm{EB}}(+0.6 \mathrm{~T},+500 \mathrm{kV} / \mathrm{m})=$ $+17.3 \mathrm{mT}, \quad \mu_{0} H_{\mathrm{EB}}(-0.6 \mathrm{~T},-500 \mathrm{kV} / \mathrm{m})=+17.5 \mathrm{mT}$, $\mu_{0} H_{\mathrm{EB}}(+0.6 \mathrm{~T},-500 \mathrm{kV} / \mathrm{m})=-19.8 \mathrm{mT}, \quad$ and $\mu_{0} H_{\mathrm{EB}}(-0.6 \mathrm{~T},+500 \mathrm{kV} / \mathrm{m})=-20.6 \mathrm{mT}$. Obviously, only the sign of $H_{\mathrm{fr}} E_{\mathrm{fr}}$ counts for the sign of $\mu_{0} H_{\mathrm{EB}}$.

Further we have tested our model by varying the strength of electric freezing field $E_{\text {fr }}$ as shown by a plot of $\mu_{0} H_{\mathrm{EB}}$ as a function of $E_{\mathrm{fr}}$ in Fig. 4. A virtually constant negative EB field, $\mu_{0} H_{\mathrm{EB}} \approx-20 \mathrm{mT}$, emerges after MEFC out of the positive remanent FM state from $T=350$ to $298 \mathrm{~K}$ for any $E_{\mathrm{fr}} \leq 0$ in various magnetic freezing fields, $\mu_{0} H_{\mathrm{fr}}=0.1$, 0.3 , and 0.6 T. Obviously, the formation of an $A$ domain in $E_{\mathrm{fr}}<0$ does not intensify the positive polarization of $S_{\mathrm{AFM}}$ compared to that obtained after MFC, i.e., in $E_{\mathrm{fr}}=0$. This situation changes drastically when applying positive freezing fields, $E_{\mathrm{fr}}>0$. At fairly low field values, $E_{\mathrm{fr}} \leq$

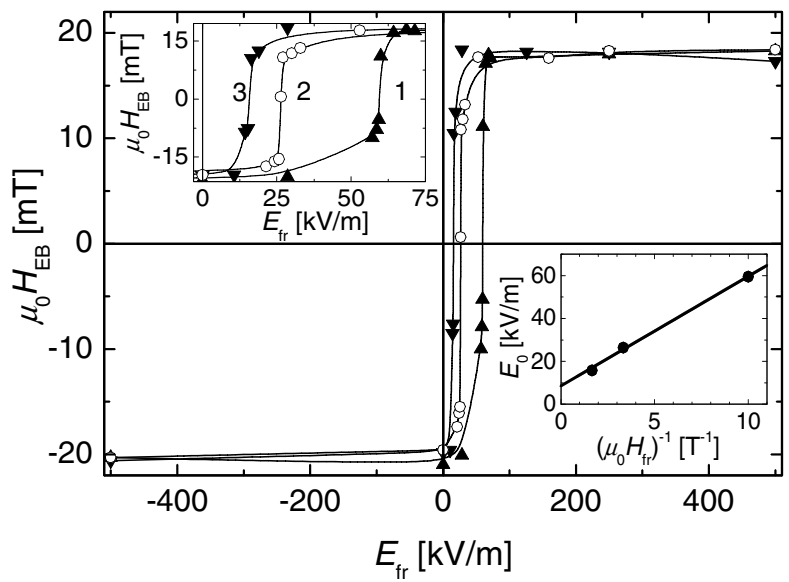

FIG. 4. Exchange bias field $\mu_{0} H_{\mathrm{EB}}$ vs electric freezing field $E_{\mathrm{fr}}$ as obtained from hysteresis loops after MEFC from $T=350$ to $298 \mathrm{~K}$ in $E_{\mathrm{fr}}$ and magnetic fields $\mu_{0} H_{\mathrm{fr}}=0.1$ (curve 1 ), 0.3 (curve 2), and $0.6 \mathrm{~T}$ (curve 3) (main panel and upper inset at enhanced scale). The lines are guides to the eye. The lower inset shows the experimental data $E_{0}$ vs $\left(\mu_{0} H_{\mathrm{fr}}\right)^{-1}$ of the electric threshold field $E_{0}=E_{\mathrm{fr}}\left(\mu_{0} H_{\mathrm{EB}}=0\right)$. The straight line indicates the best linear fit to Eq. (3).

$60 \mathrm{kV} / \mathrm{m}, \mu_{0} H_{\mathrm{EB}}$ changes from negative to positive values, which saturate at $\mu_{0} H_{\mathrm{EB}} \approx 18 \mathrm{mT}$ for $E_{\mathrm{fr}} \approx$ $100 \mathrm{kV} / \mathrm{m}$. The transition points, $E_{0}$, on the electric field axis shift to lower values as $\mu_{0} H_{\mathrm{fr}}$ is increased. This clearly hints at a competition between the two modes of field cooling: conventional MFC and MEFC as conjectured above.

In order to understand the inverse dependence of the threshold field $E_{0}$ on the magnetic freezing field, $\mu_{0} H_{\mathrm{fr}}$ (see enlarged plot of the transition curves in the upper inset in Fig. 4), we consider the energy of the AFM interfacial spins during the freezing process. First, we have to take into account the ME induced energy difference between domain types $A$ and $B, W_{\mathrm{ME}} \propto-\alpha_{\mathrm{zz}} \mu_{0} H_{\mathrm{fr}} E_{\mathrm{fr}}$. Second, the magnetostatic Zeeman energy of the AFM interface spins, $W_{\mathrm{Z}} \propto-\mu_{0} H_{\mathrm{fr}} S_{\mathrm{AFM}}$, has to be considered. Third, the exchange interaction at the FM-AFM interface yields $W_{\mathrm{EX}} \propto$ $-J S_{\mathrm{FM}} S_{\mathrm{AFM}}$, which is independent of $H_{\mathrm{fr}}$ under the assumption of a single domain FM state. Hence, $H_{\mathrm{EB}}$ will vanish, if the ME energy $W_{\mathrm{ME}}$ is compensated by the MFC contributions $W_{\mathrm{Z}}$ and $W_{\mathrm{EX}}$,

$$
W_{\mathrm{ME}}=W_{\mathrm{Z}}+W_{\mathrm{EX}}
$$

Dividing both sides in Eq. (2) by $\mu_{0} H_{\text {fr }}$ we readily obtain the electric threshold field,

$$
E_{0}=c_{1}+c_{2} / \mu_{0} H_{\mathrm{fr}}
$$

where $c_{1}$ and $c_{2}$ are fitting parameters. Indeed, as shown in the lower inset of Fig. 4 the experimental data $E_{0}$ vs 
$\left(\mu_{0} H_{\mathrm{fr}}\right)^{-1}$ reveal linearity and are best fitted by $c_{1}=8.63 \mathrm{kV} / \mathrm{m}$ and $c_{2}=5104 \mathrm{~V}^{2} \mathrm{~s} / \mathrm{m}^{3}$. The constant $c_{1}$, which formally equals $E_{0}$ in the limit $H_{\text {fr }} \rightarrow \infty$, denotes the electric field compensating the Zeeman contribution to $H_{\mathrm{EB}}$ for large $H_{\mathrm{fr}}$.

Let us finally remark that the newly discovered switching effect due to magnetoelectric single domaining at the interface is giant compared to the ME shift of $H_{\mathrm{EB}}$ when applying an electric field to the AFM single domain after MEFC. Although a sizable magnetic moment arises in the bulk (Fig. 3, lines 2 and 3), only a minute change of $S_{\mathrm{AFM}}$ has been predicted [10] and experimentally confirmed [16]. For the present sample we have observed $\left|\mu_{0} \Delta H_{\mathrm{EB}}\right| \approx$ $0.3 \mathrm{mT}$ for $|E|=500 \mathrm{kV} / \mathrm{m}$ and $T=250 \mathrm{~K}$, which remains invisible within the symbol widths of Fig. 1. Albeit being disappointingly small at the first glance, future use of all thin-film samples promises to improve the situation considerably. Similarly, it can be expected that the performance of the present switching mechanism might be further enhanced in proper thin-film samples, which then would promise applicability in future magnetoelectronic devices. For example, shifting the hysteresis loop from positive to negative magnetic fields by proper field-cooling procedures might be an interesting alternative to conventional current driven magnetic switching of spin valves from high to low resistance values and vice versa.

In summary, we investigated a new kind of EB system consisting of a FM multilayer on top of a ME AFM single crystal. By inverting the electric freezing field and thus the AFM single domain, we were able to switch the interface magnetization $S_{\mathrm{AFM}}$ and thus the EB field, $H_{\mathrm{EB}}$. This way of electrically controlling EB opens new possibilities for tailoring magnetoresistive components with very low power consumption, but simultaneously requires further research on ME EB systems. In the present case we propose single AFM unit cells to be related to the uncompensated pinned interface spins $S_{\mathrm{AFM}}$ discussed in the literature [17]. Their pinning mechanism remains unexplained. Further, it will be interesting if similar ME coupling can be realized with multiferroic materials.

Fruitful discussions with M. Fiebig and technical support by M. Aderholz are gratefully acknowledged. This research was supported by the Deutsche Forschungsgemeinschaft through SFB 491.
*Electronic address: kleemann@uni-duisburg.de

[1] B. D. H. Tellegen, Philips Res. Rep. 3, 81 (1948).

[2] T. Kimura, T. Goto, H. Shintani, T. Arima, and Y. Tokura, Nature (London) 426, 55 (2003).

[3] N. Hur, S. Park, P. A. Sharma, J. S. Ahn, and S-W. Cheong, Nature (London) 429, 392 (2004).

[4] T. Lottermoser, T. Lonkai, U. Amann, D. Hohlwein, J. Ihringer, and M. Fiebig, Nature (London) 430, 541 (2004).

[5] D. N. Astrov, Sov. Phys. JETP 11, 708 (1960); 13, 729 (1961).

[6] W.H. Meiklejohn and C.P. Bean, Phys. Rev. 102, 1413 (1956); 105, 904 (1957).

[7] G. A. Prinz, Science 282, 1660 (1998).

[8] J. Nogués and I. Schuller, J. Magn. Magn. Mater. 192, 203 (1999).

[9] Ch. Binek, X. Chen, A. Hochstrat, and W. Kleemann, J. Magn. Magn. Mater. 240, 257 (2002).

[10] A. Hochstrat, Ch. Binek, Xi. Chen, and W. Kleemann, J. Magn. Magn. Mater. 272-276, 325 (2004).

[11] B. Kagerer, Ch. Binek, and W. Kleemann, J. Magn. Magn. Mater. 217, 139 (2000); S. Maat, K. Takano, S. S.P. Parkin, and E. E. Fullerton, Phys. Rev. Lett. 87, 087202 (2001).

[12] R. Cheng, C. N. Borca, N. Pilet, Bo Xu, L. Yuan, B. Doudin, S. H. Liou, and P. A. Dowben, Appl. Phys. Lett. 81, 2109 (2002); 82, 1443 (2003).

[13] S. Shtrikman and D. Treves, Phys. Rev. 130, 986 (1963); C. A. Brown Ph.D. thesis, Imperial College, 1969; T. H. O'Dell The Electrodynamics of Magneto-Electric Media (North-Holland, Amsterdam, 1970).

[14] P. J. Brown, J. B. Forsyth, and F. Tasset, J. Phys. Condens. Matter 10, 663 (1998).

[15] T. H. O’Dell, Philos. Mag. 13, 921 (1966).

[16] Ch. Binek, A. Hochstrat, X. Chen, P. Borisov, W. Kleemann, and B. Doudin, J. Appl. Phys. (to be published); Ch. Binek, P. Borisov, Xi. Chen, A. Hochstrat, S. Sahoo, and W. Kleemann, Eur. Phys. J. B (to be published).

[17] A. E. Berkowitz and K. Takano, J. Magn. Magn. Mater. 200, 552 (1999); H. Ohldag T. J. Regan, J. Stöhr, A. Scholl, F. Nolting, J. Lüning, C. Stamm, S. Anders, and R. L. White, Phys. Rev. Lett. 87, 247201 (2001); H. Ohldag, A. Scholl, F. Nolting, E. Arenholz, S. Maat, A. T. Young, M. Carey, and J. Stöhr, Phys. Rev. Lett.91, 017203 (2003).

[18] P. Miltényi, M. Gierlings, J. Keller, B. Beschoten, G. Güntherodt, U. Nowak, and K. D. Usadel, Phys. Rev. Lett. 84, 4224 (2000). 